\title{
Heisenberg Spins on a Circular Conical Surface
}

\author{
Afranio R. Pereira \\ Departamento de Física, Universidade Federal de Viçosa, Viçosa, 36570-000 \\ Minas Gerais, Brazil.
}

\begin{abstract}
We investigate classical Heisenberg spins on a conical surface. The energy and configuration of non-trivial spin distributions are obtained using a non-conventional method based on Einstein theory of gravity in lower dimensions.

PACS numbers: 75.10. Hk; 75.10.-b; 05.45.Yv;
\end{abstract}

Keywords: Solitons; Magnetic materials; Curved surfaces.

Corresponding author: A. R. Pereira

e-mail: apereira@ufv.br

Tel.: $+55-31-3899-2988$.

Fax: +55-31-3899-2483. 
important as we move to an era of important technological realizations. Physics in two spatial dimensions has generated a lot of information and surprises. Moreover, if the system lies on a curved surface, it adds further richness to the physical phenomena [1-4]. Recently, it has become possible the fabrication of magnetic films grown on a curved substrate [5]. Researchers already discovered how to produce graphitic cones in 1997 [6,7]. In this letter we consider Heisenberg spins on a conic support. Although we do not know of physically relevant examples, it should be interesting to investigate the behavior of topological structures on exotic surfaces. After all, artificially structured materials are produced each day leading to new knowledge and technologies. Besides, the idea that topologically nontrivial excitations arise in real physical systems had a strong impact on modern physics. These structures became an object of intensive investigations in many condensed matter models and particularly they are believed to play an important role in magnetic systems $[8,12]$. Our main interest is to study the configuration and energy of such topological excitations on the surface of a cone.

As it is well known, the classical two-dimensional static ferromagnetic and antiferromagnetic materials are well described, in the continuum limit, by the non-linear $\sigma$ model. This theory on the surface of a curved geometry is [4]

$$
H=\frac{J}{2} \iint_{S}\left(D_{i} \vec{\psi}\right)\left(D^{i} \vec{\psi}\right) \sqrt{|g|} d \Omega, \quad i=1,2
$$

where $D_{i}$ is the covariant derivative, $d S=\sqrt{|g|} d \Omega$ is the surface, $\sqrt{|g|}$ is the determinant of the metric tensor $g_{i j}$ and the classical spin field $\vec{\psi}$ obeys the constraint $\vec{\psi}^{2}=\psi_{1}^{2}+\psi_{2}^{2}+\psi_{3}^{2}=1$. Here, we consider a non-conventional approach that may simplify the calculations significantly. Instead of using Hamiltonian (1) with adequate metric tensor for a cone surface and resolving it, we apply some results of general relativity. In fact, the conical geometry has connections with the problem of Einstein gravity in three space-time $(2+1)$ dimensions. This theory exhibits some unusual features, 
[13]. In regions which are free of matter, space-time is locally flat when the cosmological constant is zero [13]. Although the local curvature in regions free of sources is unaffected by any matter in space-time, there is still nontrivial global effects. The reason that these spaces are not completely trivial is because of nontrivial boundary conditions on the flat coordinates. For instance, in the case of a massive point-particle sitting at rest in the origin, we have to remove a wedge out of space-time and identify opposite points of the wedge. In the static case, the unique two-dimensional spatial geometry satisfying this description is the cone [14]. Really, the three-dimensional space-time around a point-particle of mass $M$ has an interval given by [13-15] $d s^{2}=d t^{2}-r^{-8 G M}\left(d r^{2}+r^{2} d \varphi^{2}\right)$, where $G$ is the gravitational constant and $r, \varphi$ are assumed to be the polar coordinates. Since the curvature vanishes everywhere except at the particle position, this interval can be written using local flat coordinates as $d s^{2}=d t^{2}-d \rho^{2}-\rho^{2} d \phi^{2}$, where

$$
\rho=\frac{r^{\beta}}{\beta}, \quad \phi=\beta \varphi,
$$

and $\beta=1-4 G M$. Note that although the situation looks trivial, the new coordinate $\phi$ ranges from 0 to $2 \pi \beta$, indicating that there is a deficit angle in space. Thus, it is always possible to transform to coordinates in which $g_{i j}$ becomes the Minkowski metric, but paying a price, i.e., we will have to work with multivalued coordinates (or coordinates with strange boundary conditions). For a static space-time, coordinates can be chosen for which each of the $t=$ constant spatial sections are identical. We use these results to study a Heisenberg spin system on a conic surface.

In the case in which $r, \varphi$ are the polar coordinates of a point in a two-dimensional plane, the metric tensor can be written as

$$
g_{i j}=\left[\begin{array}{cc}
1 & 0 \\
0 & r^{2}
\end{array}\right]
$$


Then, it is easy to write Hamiltonian (1) for spins in a flat metric. In order to transform this Hamiltonian on a flat space to the problem of a conical surface, we write first $r$ and $\varphi$ in terms of $\rho$ and $\phi$ respectively, using $\beta=1-\alpha$ for a space with a deficit angle equal to $2 \pi \alpha$. It would imply that the cone surface makes an angle of $\gamma=\arcsin (\beta)$ with its symmetry z-axis (this axis passes for a point on the tip of the cone). Thus, Hamiltonian (1) can be easily written on a conical geometry as

$$
H=\frac{J}{2} \int_{0}^{\infty} \int_{0}^{2 \pi \beta}\left(\partial_{i} \vec{\psi}\right)\left(\partial^{i} \vec{\psi}\right) \rho d \rho d \phi
$$

where $\partial_{i}$ is the gradient written in terms of the coordinates $(\rho, \phi)$. Now, we would like to get nonlinear excitation solutions with non-zero but finite energy on the surface of a cone. For the two-dimensional plane $(\beta=1)$, these solutions are the well known Belavin-Polyakov (BP) solitons [16]. Structurally, these excitations correspond to a mapping of the spin sphere $\left(\vec{\psi}^{2}=1\right)$ onto the physical coordinate plane with a constant field $\vec{\psi}$ far from the soliton center. Essentially, the plane is compacted into a spherical surface and $\vec{\psi}$ is a mapping of the sphere to itself classified by a topological charge $q$ that gives the number of times $\vec{\psi}$ does this. Hence, there is the Bogomol'nyi inequality [16] $H \geq 4 \pi J|q|$, where the minimum energy for a given $q$ is $4 \pi J|q|$. In terms of the conformal representation $w=\left(\psi_{1}+i \psi_{2}\right) /\left(1-\psi_{3}\right)$, which is simply the stereographic projection of the vector $\vec{\psi}$ onto the complex plane, the BP-solitons are expressed as $w_{S}=\left[\left(z-z_{0}\right) / R\right]^{n}$, where $z=x+i y$ and the constants $R, z_{0}$ appear due to scale and translational symmetry. The energy of these static structures is finite depending only upon the total topological charge $|q|=n=1,2,3, \ldots$, and is given by $4 \pi J|q|$; there is no preferred size $R$, position or orientation for a fundamental soliton.

For the conical geometry $(0<\beta<1)$, part of the spin space sphere is not included in the mapping due to the deficit angle. Now, the exigency that $\vec{\psi}$ must be constant at infinity makes that the plane be compacted into a spherical surface with a hole on it. The area of the hole $(4 \pi \alpha)$ is surrounded by two lines (part of geodesics or great circles) drawn from the top point of the sphere 
equal to $2 \pi \alpha$. However, the identification of points across the wedge will close this hole, transforming the space sphere into a spheroid (something like an oval ball of the American football) with area $4 \pi \beta$. At the same time, the spin sphere will have to become also a spin spheroid with the same shape of the space spheroid, due to the boundary conditions used after to remove a wedge from the physical space, i.e., the spins will have to obey the relation $\vec{\psi}(0)=\vec{\psi}(2 \pi \beta)$. The surface of the spin spheroid should be written in terms of a new spin field $\vec{\chi}=\left(\beta \psi_{1}, \beta \psi_{2}, \psi_{3}\right)$, since this surface is flattened at equator, which has a radius $\beta$. As a consequence, we will find an one-to-one mapping from the set of points on the spin spheroid onto the set points on the cone (stereographic projection). However, this stereographic projection is difficult to be done because the spheroid surface does not has a so simple expression. Then, instead of using the elegant topological arguments as Belavin and Polyakov did for the flat space, we will solve explicitly the nonlinear partial differential equations obtained from Hamiltonian (4). To do this, we will consider explicitly only excitations with unitary topological charge. The generalization of the arguments is direct for generic $q$. To solve this Hamiltonian problem, it is convenient to parametrize the spin field in terms of two scalar fields $\vec{\psi}=\left(\sqrt{1-m^{2}} \cos (\Phi), \sqrt{1-m^{2}} \sin (\Phi), m\right)$, where $m=\cos (\theta)$, and $\theta$ and $\Phi$ are the polar and the azimuthal angles respectively. Then, Hamiltonian (4) is expressed as

$$
H_{c}=\frac{J}{2} \int_{0}^{\infty} \int_{0}^{2 \pi \beta}\left[\frac{(\vec{\nabla} m)^{2}}{1-m^{2}}+\left(1-m^{2}\right)(\overrightarrow{\nabla \Phi})^{2}\right] \rho d \rho d \phi
$$

The equations of motion, obtained after variation of the above Hamiltonian $\delta H_{c}=0$, are given by

$$
\begin{gathered}
\nabla^{2} m+\frac{m(\vec{\nabla} m)^{2}}{1-m^{2}}+m\left(1-m^{2}\right)(\vec{\nabla} \Phi)^{2}=0 \\
\nabla^{2} \Phi-\frac{2 m \vec{\nabla} m \cdot \vec{\nabla} \Phi}{1-m^{2}}=0 .
\end{gathered}
$$


Clearly, a special solution of Eqs.(6) and (7) is the soliton $\left(m_{c}, \Phi_{c}\right)$ given by

$$
m_{c}= \pm \frac{\rho^{2}-\rho_{0}^{2}}{\rho^{2}-\rho_{0}^{2}}, \quad \Phi_{c}=\phi
$$

where $\rho_{0}$ is a constant. In analogy with the usual case of a flat space, we associate the signals + and - with configurations with topological charges equal to +1 and -1 respectively.

Now, to see the configuration of a soliton on the conical surface more clearly, it would be useful to return to variables $(r, \varphi)$. Here, since the coordinate $r$ becomes the smallest distance of a point on the cone surface from the point in which the cone narrows to, i.e., the origin of the coordinates system or the tip of the cone, the soliton structure can be written in a general way as a simple function of $\beta$. In this case, the identification $\Phi_{c}(0)$ with $\Phi_{c}(2 \pi \beta)$ is always possible for a configuration with radial symmetry. Thus, the soliton structure on a conic support is

$$
m_{c}= \pm \frac{r^{2 \beta}-R^{2 \beta}}{r^{2 \beta}+R^{2 \beta}}, \quad \Phi_{c}=\beta \varphi
$$

where $R=\beta \rho_{0}^{1 / \beta}$ is the soliton size, obtained considering the points on the conical surface in which the spins are perpendicular to the cone axis (z-axis). These points form a circumference that divide the cone into two parts with relation to spin directions: above this line, $m_{c}>0\left(m_{c}<0\right)$, while below it, $m_{c}<0\left(m_{c}>0\right)$. This soliton size can be chosen arbitrarily since the energy of this structure is

$$
E_{c}=4 \pi J \beta
$$

and does not depend on $R$. Then, such an excitation can have arbitrarily large size, thanks to the 
structure has the spin $\vec{\psi}$ pointing in all different directions as $\vec{r}$ varies, but each cone can support only one soliton, since there is a preferred position for its nucleation. In fact, the solutions obtained here cannot move on the conical surface even for antiferromagnetic systems, which are, in general, Lorentz invariant. The reasons are simple; first, the translational symmetry was broken. Second, the Lorentz transformation is a global one while the cone surface has a Minkowski metric only locally. Hence these solitons are pinned to the tip point of the cone. Curiously, pinned non-linear excitations are also possible in a flat space with vacancies. Recently, some soliton solutions pinned to a nonmagnetic impurity were obtained [8-12] and experimentally observed [8,9] in two-dimensional flat magnetic materials. In this case, part of the spin space sphere cannot be included in the mapping (around the impurity), since there is no spin on a vacancy. Essentially, in these two situations (the flat space with vacancy and the cone), solitons are pinned to a determined point due to oppositive causes: in one case, there is a missing spin while in the other, it is missing space to put spins.

The above calculations can be easily generalized to excitations with $|q|>1$, which leads to a soliton energy equal to $4 \pi J \beta|q|$. Clearly, $q$ gives the number of times the spin spheroid is traversed as we span the coordinate space as compacted into another spheroid. Of course, as expected, when $\beta \rightarrow 1, E_{c} \rightarrow 4 \pi J|q|$, which is the BP-soliton energy for solitons in the flat space. It is also important to note that Eqs. (9) and (10) are explicitly dependent on the geometry of the support (the parameter $\beta$ or $\gamma=\arcsin (\beta)$ ). While a cone does not have a characteristic length, it has a characteristic angle $\gamma$. The spin projection along the symmetry axis (z-axis) is extremely dependent on this angle. In Fig.(1), we plotted $m_{c}$ as a function of $r$ for $q= \pm 1, \beta=1,1 / 2,1 / 4$ and $R=5$ (in units of lattice constants). For small $\beta$ (i.e., a large deficit angle), $m_{c}(r)$ varies very slowly for $r>R$. Figures (2) and (3) show schematic representations of the spin configuration of a magnetic soliton with $q= \pm 1$ on a conical surface.

In summary, we have studied the Heisenberg spins on a conic support and obtained soliton solutions. It was shown that solitons can be formed on the tip of a cone. Part of the spin 
a soliton energy equal to $4 \pi J \beta|q|$. Then, the effect of removing a wedge from the flat space and identifying points across the wedge is essentially to change the BP-soliton configuration decreasing its energy. The results presented here may have relevance for the recently synthesized carbon nanocones [6] with appropriate magnetic coatings. If one or more sectors are excised from a single layer of graphite and the remainder is joined seamlessly, a cone results [6,17]. By considering the symmetry of a graphite sheet and the Euler's theorem, it can be shown that only five types of cone can be made from a continuous sheet of graphite corresponding to the following values of cone angles $2 \gamma=19.2^{\circ}, 38.9^{\circ}, 60.0^{\circ}, 84.6^{\circ}, 112.9^{\circ}$. These are all synthesized [6]. The solitons excited on the tips of these magnetically coated carbon nanocones would have energies given by $E_{c} \simeq 2.1 J, 4.2 J, 6.3 J, 8.5 J$ and 10.5J respectively. Besides the carbon nanocones, magnetic nanodots can be made of different shapes and display a variety of magnetic excitations. In the case of a magnetic dot with conical shape, the above solitons may be important for the resulting magnetization distributions. Really, in magnetic nanostructures, solitons can be stabilized by surface-induced interactions [18]. Another possibility is to study a spin-polarized electron gas on the conical surface. Such an electron system was studied on a mesoscopic cylinder [3]. Here, not only charges but also the spins may develop important role near the sharp point of the cone. These results are also relevant to field theories considered on manifolds with nontrivial geometry and have connection with lower dimensional gravity, mainly three-dimensional gravity, in which the non-linear $\sigma$ model is coupled to Einstein field.

We acknowledge support from CNPq (Brazil). 
[1] S. Villain-Guillot, R. Dandoloff, A. Saxena, Phys. Lett. A 188 (1994) 343.

[2] R. Dandoloff, S. Villain-Guillot, A. Saxena, A. R. Bishop, Phys. Rev. Lett. 74 (1995) 813.

[3] R. Dandoloff, A. Saxena, Eur. Phys. J. B 29 (2002) 265.

[4] A. Saxena, R. Dandoloff, Phys. Rev. B 55 (1997) 11049.

[5] R.K. Kawakami, E.J. Escorcia-Aparicio, Z.Q. Qiu, Phys. Rev. Lett. 77 (1996) 2570.

[6] A. Krishnan, E. Dujardin, M.M.J. Treacy, J. Hugdahl, S. Lynum, T.W. Ebbesen, Nature (London) 388 (1997) 451.

[7] M. Ge, K. Sattler, Chem. Phys. Lett. 220 (1994) 192.

[8] K. Subbaraman, C.E. Zaspel, J.E. Drumheller, Phys. Rev. Lett. 80 (1998) 2201.

[9] C.E. Zaspel, J.E. Drumheller, K. Subbaraman, Phys. Status Solidi A 189 (2002) 1029.

[10] A.R. Pereira, A.S.T. Pires, J. Magn. Magn. Mater. 257 (2003) 290.

[11] L.A.S. Mól, A.R. Pereira, W.A. Moura-Melo, Phys. Rev. B 67 (2003) 132403.

[12] A.R. Pereira, Phys. Lett. A 314 (2003) 102.

[13] J.D. Brown, Lower Dimensional Gravity (World Scientific, New Jersey, 1988), and references therein.

[14] A. Staruszkiewicz, Acta. Phys. Polon. 24 (1963) 734.

[15] M. Welling, arXiv:hep-th/9511211 (1995).

[16] A.A. Belavin, A.M. Polyakov, JEPT Lett. 22 (1975) 245.

[17] P.E. Lammert, V.H. Crespi, Phys. Rev. Lett. 85 (2000) 5190.

[18] A. N. Bogdanov, U.K. Röbler, Phys. Rev. Lett. 87 (2001) 037203. 
Figure 1. The z-spin components $m_{c}$ of a soliton with $q=1$ on a conical surface versus $r$ for $\beta=1$ (solid line), $\beta=1 / 2$ (dashed line) and $\beta=1 / 4$ (dotted line). All these solitons have the same size $R=5$ (in units of lattice constants)

Figure 2. Schematic representation of a magnetic soliton with $q=+1$ on the conic support.

Figure 3. Schematic representation of a magnetic soliton with $q=-1$ on the conic support. 